\title{
THE APPLICABILITY OF LATTICE THEORY TO GROUP THEORY*
}

\author{
REINHOLD BAER
}

The influence of a more general theory, such as the theory of lattices, upon a more specific theory, such as the theory of groups, may make itself felt in two directions: either one tries to generalize the known theorems of group theory in order to find their place in the more general framework of lattice theory, or else one tries to use the methods of lattice theory for the solution of problems in the theory of groups which this theory has not yet been able to solve by its own means. It is the latter kind of problem that interests us in this note.

But before attacking a problem like this, one may very well ask whether or not this problem has a solution, that is, whether we have any reason to expect that the methods of lattice theory may yield a solution of our problems, and this question of the applicability of lattice theory to group theory will occupy us in the course of this investigation.

The fundamental problem of group theory is the so-called structure problem. Here I use the term "structure" in the customary sense that two groups have the same structure if they are isomorphic. This problem has been solved for rather restricted classes of groups only, notably the finite abelian groups. In some of these cases it has actually been possible to rephrase the results in terms of lattice theory, whereas in other cases no such attempt has been made. The question is now whether group theory may expect from lattice theory the answer to this as yet unsolved problem, and it is a systematic attempt to find this out which gave rise to the following considerations.

In the specific case which we are discussing, we may state as our object a delineation of the extent to which the structure of a group is determined by the structure of its lattice of subgroups. The first observation one makes, as soon as one tries to put this on a more precise basis, is that of the wealth of obvious counterexamples which seem to indicate that, as far as our problem is concerned, we may not expect much help from lattice theory. Thus I noticed with much surprise that all these counterexamples are comparatively "small"

* This paper is a slight elaboration of the author's discussion at the Symposium on Lattice Theory in Charlottesville. A more complete treatment of the topics mentioned here will be found in the author's paper The significance of lattice theory for group theory, which will appear in the American Journal of Mathematics. 
groups and that the situation seems to be quite different with the "big" groups, a state of affairs which seems to bear some resemblance to certain questions in the fundamentals of geometry.

In the following fashion one will be led to concepts which link lattice theory and group theory in a systematic way. If $G$ and $H$ are groups, and if $f$ is an isomorphism of $G$ upon (the whole group) $H$, then $f$ maps every subgroup $S$ of $G$ upon a uniquely determined subgroup $S^{f}$ of $H$. The isomorphism $f$ thus induces a correspondence between the two lattices of subgroups, and this correspondence has the following properties:

(1) The correspondence is a one-one correspondence between the whole lattice of subgroups of $G$ and the whole lattice of subgroups of $H$.

(2) $S<T$ if, and only if, $S^{f}<T^{f}$.

(3) If $S<T$, then $[T: S]=\left[T^{f}: S^{f}\right]$.

(4) The subgroups $R$ and $S$ of the subgroup $T$ of $G$ are conjugate in $T$ if, and only if, $R^{f}$ and $S^{f}$ are conjugate in $T^{f}$.

Since the properties (1) and (2) refer to lattice properties only, we shall call any correspondence which satisfies (1) and (2) a subgroupisomorphism of $G$ upon $H$. A subgroup-isomorphism will be called index-preserving, if it satisfies (3); it will be called normal, if it satisfies (4).

It may be noted here that it is often sufficient to assume instead of (3) and (4) the following weaker conditions:

$\left(3^{\prime}\right)$ If $T$ is a cyclic subgroup of $G$ and $S<T$, then $[T: S]=\left[T^{f}: S^{f}\right]$.

(4') $S$ is a normal subgroup of $G$ if, and only if, $S^{f}$ is a normal subgroup of $H$.

The problem of whether or not lattice theory will furnish the answer to the structure problem of some group $G$ may now be stated in this form:

Is it true that every group is isomorphic to $G$ which is subgroupisomorphic to $G$ ?

An affirmative answer to this question would reduce the structure theory of the group $G$ to a problem in lattice theory.

As the "exterior structure theory" of the group $G$ characterizes those groups which are isomorphic to $G$, so the "interior structure theory" of $G$ characterizes those pairs of subgroups $S, T$ of $G$ for which there exists an automorphism of $G$ mapping $S$ upon $T$. If one wants to reduce both the exterior and the interior structure theory 
of the group $G$ to a problem in lattice theory, then one has to prove a statement like the following one: Every subgroup-isomorphism of the group $G$ is induced by an isomorphism of $G$. And this last problem finally is a special case of the question as to which subgroup-isomorphisms are induced by isomorphisms proper.

The history of these problems dates back to 1928, that is, five years before the modern revival of lattice theory. At that time E. Fischer noted that the fundamental theorem of the theory of Galois stated nothing but the existence of an isomorphism between the lattice of the subgroups of the Galois group and a certain lattice of fields and that this isomorphism satisfies conditions analogous to the above conditions of normality and index-preservation. He consequently proposed the problem of determining whether or not two groups which are connected by normal and index-preserving subgroup-isomorphisms would be from necessity isomorphic in the customary sense of the word. A. Rottlaender, a pupil of his, answered this question in the negative by proving the existence of a normal and index-preserving subgroup-isomorphism between two finite nonabelian and nonisomorphic groups. Rottlaender showed furthermore that there exist index-preserving subgroup-isomorphisms between abelian groups of order $p^{3}$ and nonabelian groups. It is finally an obvious remark that any two groups of order a prime number are subgroup-isomorphic; and it has been shown that every noncyclic group of order $p^{2}$ where $p$ is a prime number, neither 2 nor 3 , possesses subgroup-automorphisms which are normal and index-preserving but which are not induced by automorphisms of the group.

So far this is the story of adversity. However there are more positive results which give quite a different picture and seem to indicate that, as has been remarked, all these counterexamples are "too small." But the existence of these "small" counterexamples complicates the proofs, since every "greater" group might contain some of the "smaller" ones as subgroups or quotient groups; and even the proof of as simple a statement as the fact that any group which is subgroup-isomorphic to an infinite cyclic group is itself an infinite cyclic group is not trivial.

The following is a short survey of known results:*

THEOREM A. If the group $G$ is either a symmetric or an alternating group, and if $f$ is an index-preserving subgroup-isomorphism of $G$ upon the group $H$ which satisfies the normality condition (4'), then $G$ and $H$ are isomorphic.

\footnotetext{
* For proofs see the author's paper, mentioned in the first footnote of this paper.
} 
THEOREM B. If $f$ is an index-preserving subgroup-isomorphism of the abelian group $G$ upon the abelian group $H$, then $G$ and $H$ are isomorphic groups.

Theorem $\mathrm{B}$ admits of some refinements, but it would lead us too far to enumerate them here.

THEOREM C. The subgroup-isomorphism $f$ of the group $G$ is induced by an isomorphism of the group $G$, if at least one of the following conditions is satisfied:

(a) $G$ is a primary Hamiltonian group.

(b) $G$ is an abelian group which contains at least two independent elements of infinite order.

(c) $G$ is an abelian group without elements of infinite order so that $G$ either does not contain elements of order $n$ at all or contains at least three independent ones, and so that $G$ contains elements of prime number order $p$ if, and only if, $G$ contains elements of order $p^{2}$.

That these conditions are "best," as far as abelian groups are concerned, may be seen from suitable examples.

Finally I would like to mention some open problems which seem to be promising and of importance in this context:

I. To enumerate all the groups which are subgroup-isomorphic to abelian groups.

II. To discuss the subgroup-isomorphisms of simple groups and of their direct products.

III. To decide whether or not free groups and free products are characterized by their lattices of subgroups.

This last problem seems particularly important, since for the decomposition of a group into free factors a completely unrestricted refinement (and uniqueness) theorem* holds.

UNIVERSITY OF ILLINOIS

* Cf. R. Baer and F. Levi, Freie Produkte und ihre Untergruppen, Compositio Mathematica, vol. 3 (1936), pp. 391-398. 